\title{
GROUPS OF MOTIONS AND MINIMAL IMMERSIONS OF SPHERES INTO SPHERES
}

\author{
Dedicated to Professor Kentaro Yano on his 75th birthday
}

By Yosio Mutō

\section{Introduction.}

Let $f$ be an isometric minimal immersion: $S^{m}(1) \rightarrow S^{n-1}(r)$ and let $g$ be a rotation: $S^{m}(1) \rightarrow S^{m}(1)$. Then $f \circ g: S^{m}(1) \rightarrow S^{n-1}(r)$ is also an isometric minimal immersion. Though the point set $f \circ g\left(S^{m}(1)\right)$ is equal to the point set $f\left(S^{m}(1)\right)$, $f \circ g$ is not in general the same with $f$. If $f \circ g$ is equivalent to $f$ in the sense of do Carmo and Wallach [1], then we say that $f$ is a $g$-invariant immersion. In general there exist isometric minimal immersions which are not $g$-invariant if $m \geqq 3$.

Let $K$ be a skew $(m+1) \times(m+1)$ matrix of $R^{m+1}$ in which $S^{m}(1)$ is embedded as the unit hypersphere and $k$ be the one-parameter subgroup of $\mathrm{SO}(m+1)$ generated by $K$, so that $k(t)=e^{K t}$. If, for every $t, f \circ k(t)$ is equivalent to $f$, we say that $f$ is $K$-invariant or $k$-invariant. The element $C$ of $W_{2}$ (see [2], [3]) associated with $f$ is then said to be $K$-invariant or $k$-invariant.

This notion can be extended as follows.

Let $K_{1}, \cdots, K_{p}$ be skew $(m+1) \times(m+1)$ matrices and $k$ be the subgroup of $\mathrm{SO}(m+1)$ generated by $K_{1}, \cdots, K_{p}$. If $C$ is invariant by $K_{1}, \cdots, K_{p}, C$ is said to be $\left(K_{1}, \cdots, K_{p}\right)$-invariant or $k$-invariant.

Then there arise, for example, the following problems.

$(\alpha)$ Find all continuous subgroups $k$ of $\mathrm{SO}(m+1)$ such that there exist nontrivial $k$-invariant elements of $W_{2}$.

( $\beta$ ) Find the set $\beta$ of elements $C$ of $W_{2}$ such that, if $C$ is an element of (5, then there exists a non-trivial continuous subgroup of $\mathrm{SO}(m+1)$ which leaves $C$ invariant.

( $\gamma$ ) When a subgroup $k$ of $\mathrm{SO}(m+1)$ is given, find all $k$-invariant elements $C$ of $W_{2}$.

$(\boldsymbol{\delta})$ When an element $C$ of $W_{2}$ is given, find all subgroups $k$ of $\mathrm{SO}(m+1)$ such that $C$ is $k$-invariant.

The purpose of the present paper is, on the one hand, to study such problems in some easier cases. On the other hand the paper contains some theorems on geodesics. Geodesics in minimal immersions were studied by some authors.

Received September 24, 1985 
For example, it has been reported by K. Tsukada that a standard minimal immersion, namely an isometric minimal immersion such that the corresponding $C$ vanishes, is a helical immersion [5], [6], that is, an immersion such that every geodesic has constant curvatures which do not depend on the choice of the geodesic. In general a geodesic in a non-standard isometric minimal immersion has curvatures which are not constant and depend on the choice of the geodesic. But, in some isometric minimal immersions all geodesics have constant curvatures and there exists some set of geodesics such that each member of the set has constant curvatures which are independent of the choice of the member.

First we recollect the general theory of isometric minimal immersions such as was given in [1], [2], [3], [4]. In $\S 2 K$-invariant elements $C$ of $W_{2}$ are defined and their property is studied. In $\$ 3 k$-invariant isometric minimal immersions are studied. $\S 4$ is devoted to isometric minimal immersions of $S^{3}$ into $S^{24}$. First, some results in [3] are recollected and then invariant elements of $W_{2}(3,4)$ are studied. In $\S 5$ some special elements of $W_{2}$ with $m=3, s>4$ are studied. We define there contraction of elements of $W_{2}$ and the effect of contraction is studied. $\S 6$ is devoted to the property of geodesics in the case $S^{3} \rightarrow S^{24}$.

Isometric minimal immersions $f$ of spheres into spheres in general cases were studied by M.P. do Carmo and N. R. Wallach [1]. Guided by this study the present author also studied the same subject in the form $f: S^{m}(1) \rightarrow S^{n-1}(r)$ using a different method and found that there exists a linear space $D_{s, s}^{m}$ of some bi-symmetric harmonic tensors of bi-degree $(s, s)[2],[4]$. As this space is essentially the space $W_{2}$ of do Carmo and Wallach, we prefer the notation $W_{2}(m, s)$ to $D_{s, s}^{m}$. where

We consider isometric minimal immersions $f$ of the form $S^{m}(1) \rightarrow S^{n-1}(r)$

$$
n=(2 s+m-1)(s+m-2) ! /(s !(m-1) !)
$$

and the radius $r$ satisfies

$$
r^{2}=m /(s(s+m-1))
$$

As the number $s$ plays an important role, the immersion may be denoted by $f_{s}$. $S^{m}(1)$ is considered as the unit hypersphere of $R^{m+1}$ where an orthonormal basis $\left\{e_{1}, \cdots, e_{m+1}\right\}$ is fixed and $S^{n-1}(r)$ is considered as a hypersphere with center at the origin of $R^{n}$ where an orthonormal basis $\left\{\tilde{e}_{1}, \cdots, \tilde{e}_{n}\right\}$ is fixed. Thus $f_{s}$ is given by $n$ homogeneous harmonic polynomials $f^{A}(A=1, \cdots, n)$ of degree $s$, so that, if $x$ is a point of $S^{m}(1)$ and $u(x)=u^{2}(x) e_{2}$ is the position vector in $R^{m+1}$ of $x$, then we have $i \circ f_{s}(x)=f^{A}(u(x)) \tilde{e}_{A}$, where $i$ is the isometric embedding $i: S^{n-1}(r) \rightarrow R^{n}$.

From the polynomials $f^{A}$ we get symmetric tensors $F^{A}$ of degree $s$ in $R^{m+1}$ satisfying $f^{A}(u)=F^{A}(u, \cdots, u)$ and

$$
\Sigma_{\imath} F^{A}\left(e_{\imath}, e_{\imath}, v, \cdots, v\right)=0,
$$


where $\Sigma_{\imath}$ stands for $\sum_{i=1}^{m+1}$ and $v$ is an arbitrary vector of $R^{m+1} . F^{A}$ are called tensors of degree $s$ associated with $f_{s}$.

From the tensors $F^{A}$ we defined in [2] a tensor $f_{s, s}$ of degree $2 s$ by

$$
f_{s, s}=\Sigma_{A} F^{A} \otimes F^{A}
$$

where $\Sigma_{A}$ stands for $\sum_{A=1}^{n}$.

As a special case we have a standard minimal immersion $h_{s}$. In this special case $F^{A}$ is denoted by $H^{A}$ and $f_{s, s}$ by $h_{s, s}$, hence

$$
h_{s, s}=\Sigma_{A} H^{A} \otimes H^{A} \text {. }
$$

The tensors $F^{A}$ and $H^{A}$ depend on the choice of the orthonormal basis $\left\{\tilde{e}_{1}, \cdots, \tilde{e}_{n}\right\}$, but $f_{s, s}$ and $h_{s, s}$ do not. Moreover, if $f_{s}$ and $f_{s}^{\prime}$ belong to the same equivalence class in the sense of do Carmo and Wallach, then $f_{s, s}=f_{s, s}^{\prime}$ [2]. Hence there exists only one $h_{s, s}$.

In [2] the role of the tensor $C$ defined by

$$
C=f_{s, s}-h_{s, s}
$$

is given. If we take all isometric minimal immersions $f_{s}$ and all numbers $t \in R$, then $t\left(f_{s, s}-h_{s, s}\right)$ fill a linear space now denoted by $W_{2}(m, s)$. As there exists only one $h_{s, s}$, the tensor $C$ is called the element of $W_{2}(m, s)$ associated with the given immersion $f_{s}$. When an element $C$ of $W_{2}(m, s)$ is taken arbitrarily, it may happen that there exist no $f_{s}$ satisfying (1.3). But taking a number $t$ suitably, we have $f_{s}$ satisfying $t C=f_{s, s}-h_{s, s}$. Any such $f_{s}$ is called an immersion subject to $C$.

A necessary and sufficient condition for a bi-symmetric tensor $C$ of bi-degree $(s, s)$ to be an element of $W_{2}(m, s)$ is that $C$ satisfies for any vectors $a, b$ of $R^{m+1}$

$$
\begin{gathered}
\sum_{i} C\left(e_{\imath}, e_{\imath}, a, \cdots, a ; b, \cdots, b\right)=0, \\
C(a, a, b, \cdots, b ; b, \cdots, b)=0 .
\end{gathered}
$$

Definition 1.1. When $C$ is an element of $W_{2}(m, s)$ we define $C(p, q)$ as the function $C(p, q): R^{m+1} \times R^{m+1} \rightarrow R$ such that

$$
a \times b \mapsto C(a, \cdots, a, b, \cdots, b ; a, \cdots, a, b, \cdots, b)
$$

where in the right hand side $a$ appears $p$ times before the semicolon and $q$ times after the semicolon.

As $C$ is bi-symmetric $C$ is determined when the function $C(s, 0)$ is given, namely, if $C_{1}$ and $C_{2}$ are elements of $W_{2}(m, s)$ such that $C_{1}(a, \cdots, a ; b, \cdots, b)$ $=C_{2}(a, \cdots, a ; b, \cdots, b)$ for arbitrary vectors $a, b$, then $C_{1}=C_{2}$.

Definition 1.2. Let $C$ be an element of $W_{2}(m, s)$ and $g$ be an element of $\mathrm{SO}(m+1)$. Then a bi-symmetric tensor $A$ is determined by 


$$
A(a, \cdots, a ; b, \cdots, b)=C\left(g^{-1} a, \cdots, g^{-1} a ; g^{-1} b, \cdots, g^{-1} b\right)
$$

and, as it is easy to verify, $A$ belongs to $W_{2}(m, s) . A$ is called the transform of $C$ by $g$ and is denoted by $g C$.

Let $\langle$,$\rangle be the ordinary inner product of vectors or of tensors of the same$ degree. If $C_{1}$ and $C_{2}$ are elements of $W_{2}(m, s)$, then we have $\left\langle g C_{1}, g C_{2}\right\rangle$ $=\left\langle C_{1}, C_{2}\right\rangle[3]$.

Definition 1.3. If $g C=C$ we say that $C$ is $g$-invariant.

\section{K-invariant elements of $W_{2}(m, s)$.}

Let $K$ be a skew $(m+1) \times(m+1)$ matrix, namely, an element of the Lie algebra of $\mathrm{SO}(m+1), k$ be the one-parameter subgroup of $\mathrm{SO}(m+1)$ generated by $K$ and put $k(t)=e^{K t}$.

DEFinition 2.1. If $C \in W_{2}(m, s)$ is $k$-invariant, that is, $k(t)$-invariant for every $t$, we say that $C$ is $K$-invariant.

Theorem 2.1. An element $C$ of $W_{2}(m, s)$ is $K$-invariant if and only if $C$ satisfies, for every vectors $a, b$ of $R^{m+1}$,

$$
C(K a, a, \cdots, a ; b, \cdots, b)+C(a, \cdots, a ; K b, b, \cdots, b)=0 .
$$

Proof. If $C$ is $K$-invariant, we have

$$
C\left(e^{K t} a, \cdots, e^{K t} a ; e^{K t} b, \cdots, e^{K t} b\right)=C(a, \cdots, a ; b, \cdots, b) .
$$

Differentiating with respect to $t$ and putting $t=0$, we get (2.1). If (2.1) is satisfied for arbitrary vectors $a, b$, we have, replacing $a$ and $b$ with $e^{K t} a$ and $e^{K t} b$,

hence

$$
\begin{aligned}
& C\left(K e^{K t} a, e^{K t} a, \cdots, e^{K t} a ; e^{K t} b, \cdots, e^{K t} b\right) \\
& \quad+C\left(e^{K t} a, \cdots, e^{K t} a ; K e^{K t} b, e^{K t} b, \cdots, e^{K t} b\right)=0,
\end{aligned}
$$

$$
(d / d t) C\left(e^{K t} a, \cdots, e^{K t} a ; e^{K t} b, \cdots, e^{K t} b\right)=0 .
$$

Thus we get (2.2).

As we have $g k g^{-1} g C=g k C=g C$ if $C$ is $k$-invariant, we have the following theorem.

THEOREM 2.2. Let $C$ be a k-invariant element of $W_{2}(m, s)$ and $g$ be an element of $\mathrm{SO}(m+1)$. Then the transform $g C$ is $\mathrm{gkg}^{-1}$-invariant.

The following theorem gives a way of constructing a $K$-invariant element of $W_{2}(m, s)$ in some cases. 
THEOREM 2.3. Let $C$ be any element of $W_{2}(m, s)$ and $K$ be such that the one-parameter subgroup $k$ generated by $K$ has $\tau$ as a period so that $e^{K \tau}=1$. Then $C_{K}$ defined by

$$
\tau C_{K}(a, \cdots, a ; b, \cdots, b)=\int_{0}^{\tau} C\left(e^{K t} a, \cdots, e^{K t} a ; e^{K t} b, \cdots, e^{K t} b\right) d t
$$

is a K-invariant element of $W_{2}(m, s)$.

Proof. The integrand is $\left(e^{-K t} C\right)(a, \cdots, a ; b, \cdots, b)$ where $e^{-K t} C$ belongs to $W_{2}(m, s)$. Hence $C_{K}$ belongs to $W_{2}(m, s)$. On the other hand we have, for any number $u$,

$$
\begin{aligned}
& \tau e^{K u} C_{K}(a, \cdots, a ; b, \cdots, b) \\
= & \tau C_{K}\left(e^{-K u} a, \cdots, e^{-K u} a ; e^{-K u} b, \cdots, e^{-K u} b\right) \\
= & \int_{0}^{\tau} C\left(e^{K(t-u)} a, \cdots, e^{K(t-u)} a ; e^{K(t-u)} b, \cdots, e^{K(t-u)} b\right) d t \\
= & \int_{0}^{\tau} C\left(e^{K t} a, \cdots, e^{K t} a ; e^{K t} b, \cdots, e^{K t} b\right) d t
\end{aligned}
$$

as $e^{K t}$ has period $\tau$.

It may happen that $C_{K}$ vanishes.

\section{Invariant minimal immersions.}

Any element $B$ of the space $B_{s, s}$ of bi-symmetric harmonic tensors of bi-degree $(s, s)$ is determined when the function $B(s, 0): R^{m+1} \times R^{m+1} \rightarrow R$ such that $B(s, 0): a \times b \mapsto B(a, \cdots, a ; b, \cdots, b)$ is given. The element $U$ of $B_{s, s}$ defined by

$$
\begin{aligned}
& U(a, \cdots, a ; b, \cdots, b) \\
= & \langle a, b\rangle^{s}+a_{1}\langle a, b\rangle^{s-2}\langle a, a\rangle\langle b, b\rangle+\cdots+a_{\sigma}\langle a, b\rangle^{s-2 \sigma}\langle a, a\rangle^{\sigma}\langle b, b\rangle^{\sigma}
\end{aligned}
$$

where $\sigma=[s / 2]$ is the largest integer satisfying $s-2 \sigma \geqq 0$ and $a_{1}, \cdots, a_{\sigma}$ satisfy $a_{0}=1$ and

$$
(s-2 p+2)(s-2 p+1) a_{p-1}+2 p(2 s+m-2 p-1) a_{p}=0 \quad p=1, \cdots, \sigma
$$

acts as the unit element in $B_{s, s}$ [2]. $U$ is expressed in terms of $H^{A}$ by

$$
U=(1 / \hat{c}) \Sigma_{A} H^{A} \otimes H^{A}
$$

where $c^{\prime}=r^{2} /\left(1+a_{1}+\cdots+a_{\sigma}\right)$.

In view of (3.1) and

$$
\left\langle e^{K t} a, e^{K t} b\right\rangle=\langle a, b\rangle
$$

which is valid for any vectors $a, b$ of $R^{m+1}$, we have 


$$
U\left(e^{K t} a, \cdots, e^{K t} a ; e^{K t} b, \cdots, e^{K t} b\right)=U(a, \cdots, a ; b, \cdots, b) .
$$

This shows the invariant property of $U$, which in fact follows naturally from the uniqueness of the unit element.

Let $f_{s}$ be a full isometric minimal immersion and $C$ be the element of $W_{2}(m, s)$ given by (1.3), namely, $C=\Sigma_{A} F^{A} \otimes F^{A}-\Sigma_{A} H^{A} \otimes H^{A}$ where $F^{A}$ are tensors associated with $f_{s}$. When $a$ is given let us denote $k(t) a$ by $a(t)$, hence $a=a(0)$. As we let the vector $a$ move freely on $S^{m}(1)$, each of $F^{A}(a(t), \cdots, a(t))$ determines a tensor $F^{A}(t)$ such that $F^{A}(a(t), \cdots, a(t))=F^{A}(t)(a, \cdots, a)$ and $F^{A}=$ $F^{A}(0)$. As $k(t)$ is an orthogonal matrix, $F^{A}(t)$ are harmonic and are the tensors associated with an isometric minimal immersion $f_{s}(t): S^{m}(1) \rightarrow S^{n-1}(r)$ such that $f_{s}(t): x \mapsto f_{s}(k(t) u(x))$, which we can write $f_{s}(t)=f_{s}(0) \circ k(t)$. As $U$ is $K$-invariant, $\Sigma_{A} H^{A} \otimes H^{A}$ is also $K$-invariant, and we have

$$
\begin{aligned}
& \Sigma_{A} F^{A}(t) \otimes F^{A}(t)-\Sigma_{A} H^{A} \otimes H^{A} \\
= & \Sigma_{A} F^{A}(t) \otimes F^{A}(t)-\Sigma_{A} H^{A}(t) \otimes H^{A}(t) \\
= & k(-t) C .
\end{aligned}
$$

Though the image of $S^{m}(1)$ by $i \circ f_{s}(t)$ is, as a point set, the same with the image of $S^{m}(1)$ by $i \circ f_{s}(0), f_{s}(t)$ is not in general the same with $f_{s}(0)$. But, if $C$ is $K$-invariant, namely $k(t) C=C$, then $f_{s}(t)$ is equivalent to $f_{s}(0)$ in the sense of do Carmo and Wallach. In this case there exists one and only one isometry $T(t)$ of $S^{n-1}(r)$ such that $f_{s}(t)=T(t) f_{s}(0)$ as $f_{s}$ is full. As we have $f_{s}\left(t_{1}+t_{2}\right)$ $=f_{s}(0) k\left(t_{1}+t_{2}\right)=f_{s}(0) k\left(t_{1}\right) k\left(t_{2}\right)=f_{s}\left(t_{1}\right) k\left(t_{2}\right)$, we get

$$
T\left(t_{1}+t_{2}\right) f_{s}(0)=T\left(t_{1}\right) f_{s}\left(t_{2}\right)=T\left(t_{1}\right) T\left(t_{2}\right) f_{s}(0) .
$$

Thus we can put $T(t)=e^{T t}$ and get

$$
f_{s}\left(t_{1}\right) \exp \left(K t_{2}\right)=\exp \left(T t_{2}\right) f_{s}\left(t_{1}\right) .
$$

If $f$ is an immersion subject to $C$ and $\hat{R}$ is an element of $\mathrm{SO}(n)$, then $\hat{R} f=f^{\prime}$ is also an immersion subject to $C$. If $C$ is $K$-invariant and $f$ is full, $f^{\prime}$ is also full. Then there exist only one $T$ and only one $T^{\prime}$ satisfying $f \circ e^{K t}$ $=e^{T t} \circ f, f^{\prime} \circ e^{K t}=e^{T \prime}{ }^{\prime} \circ f^{\prime}$, hence

$$
e^{T^{\prime} t}=\hat{R} e^{T t} \hat{R}^{-1}
$$

Thus we have the following theorem.

THEOREM 3.1. Let $K$ be a skew $(m+1) \times(m+1)$ matrix and $C$ be $a$ $K$-invariant element of $W_{2}(m, s)$. Then for each full isometric minimal immersion $f$ subject to $C$ there exists a skew $n \times n$ matrix $T(f)$ satisfying

and

$$
f \circ e^{K t}=e^{T(f) t} \circ f
$$

$$
\hat{R} \exp (T(f) t) \hat{R}^{-1}=\exp (T(\hat{R} f) t)
$$


where $\hat{R}$ is any element of $\mathrm{SO}(n)$.

\section{Isometric minimal immersions of $S^{3}$ into $S^{24}$.}

We recollect some results from [3]. Let us fix an orthonormal basis $\left\{e_{1}, e_{2}, e_{3}, e_{4}\right\}$ in $R^{4}$. On the other hand, we take a rectangular coordinate system in $R^{3}$ and express a point $p$ of $R^{3}$ by $p=(x, y, z)$. If we take linear transformations $J_{p}=x J_{1}+y J_{2}+z J_{3}$ defined by

$$
\begin{aligned}
& J_{p} e_{1}=-x e_{2}+y e_{3}-z e_{4}, \\
& J_{p} e_{2}=x e_{1}-y e_{4}-z e_{3}, \\
& J_{p} e_{3}=-x e_{4}-y e_{1}+z e_{2}, \\
& J_{p} e_{4}=x e_{3}+y e_{2}+z e_{1},
\end{aligned}
$$

then $J_{p}$ is an orthogonal transformation when $p$ is a point of the unit sphere $S^{2}(1)$ of $R^{3}$. $J_{1}, J_{2}, J_{3}$ satisfy the well-known formula, $J_{2} J_{3}=-J_{3} J_{2}=J_{1}, J_{3} J_{1}=$ $-J_{1} J_{3}=J_{2}, J_{1} J_{2}=-J_{2} J_{1}=J_{3}$.

Similarly, let $I_{p}=x I_{1}+y I_{2}+z I_{3}$ be defined by

$$
\begin{aligned}
& I_{p} e_{1}=-x e_{2}+y e_{3}+z e_{4}, \\
& I_{p} e_{2}=x e_{1}+y e_{4}-z e_{3}, \\
& I_{p} e_{3}=x e_{4}-y e_{1}+z e_{2}, \\
& I_{p} e_{4}=-x e_{3}-y e_{2}-z e_{1} .
\end{aligned}
$$

Then $I_{p}$ is an orthogonal transformation when $p$ is a point of the unit sphere and $I_{1}, I_{2}, I_{3}$ satisfy $I_{2} I_{3}=-I_{3} I_{2}=I_{1}, I_{3} I_{1}=-I_{1} I_{3}=I_{2}, I_{1} I_{2}=-I_{2} I_{1}=I_{3}$. Moreover we have

$$
\begin{gathered}
J_{\kappa} I_{\lambda}=I_{\lambda} J_{\kappa}, \\
\sum_{i}\left\langle J_{\kappa} e_{\imath}, I_{\lambda} e_{\imath}\right\rangle=0
\end{gathered}
$$

where, here and in the sequel, we use indices $\kappa, \lambda, \mu, \nu=1,2,3$.

Denoting the identity transformation in $R^{4}$ by $J_{0}$ and also by $I_{0}$, we have

$$
\begin{aligned}
& J_{\mu} J_{\lambda}+J_{\lambda} J_{\mu}=-2 \delta_{\mu \lambda} J_{0}, \\
& I_{\mu} I_{\lambda}+I_{\lambda} I_{\mu}=-2 \delta_{\mu \lambda} I_{0} .
\end{aligned}
$$

Thus $\left\{a I_{0}+b I_{1}+c I_{2}+d I_{3}, a^{2}+b^{2}+c^{2}+d^{2}=1\right\}$ is a subgroup of $\mathrm{SO}(4)$ which we write $O_{I}$ and $\left\{a J_{0}+b J_{1}+c J_{2}+d J_{3}, a^{2}+b^{2}+c^{2}+d^{2}=1\right\}$ is a subgroup of $\mathrm{SO}(4)$ which we write $O_{J} . O_{I}$ and $O_{J}$ commute and generate $\mathrm{SO}(4)$.

In what follows we denote the point $p$ by $\left(x^{1}, x^{2}, x^{3}\right)$. A homogeneous harmonic polynomial $A(x)$ in $R^{3}$ of degree 4 can be written $A(x)=A_{\kappa \lambda \mu \nu} x^{\kappa} x^{\lambda} x^{\mu} x^{\nu}$ or $A(x)=A^{\kappa \lambda \mu \nu} x^{\kappa} x^{\lambda} x^{\mu} x^{\nu} . \quad A_{\kappa \lambda \mu \nu}$ are symmetric and satisfy 


$$
\sum_{\kappa} A_{\kappa \kappa \mu \nu}=0 \text {. }
$$

Definition 4.1. The bi-symmetric tensor $C$ given by

$$
(C(4,0))(a, b)=A^{\kappa \lambda \mu \nu}\left\langle J_{\kappa} b, a\right\rangle\left\langle J_{\lambda} b, a\right\rangle\left\langle J_{\mu} b, a\right\rangle\left\langle J_{\nu} b, a\right\rangle
$$

is an element of $W_{2}(3,4)$ and is denoted by $C_{J}^{(A)}$. Similarly the bi-symmetric tensor $C$ given by

$$
(C(4,0))(a, b)=B^{\kappa \lambda \mu \nu}\left\langle I_{\kappa} b, a\right\rangle\left\langle I_{\lambda} b, a\right\rangle\left\langle I_{\mu} b, a\right\rangle\left\langle I_{\nu} b, a\right\rangle
$$

is an element of $W_{2}(3,4)$ and is denoted by $C_{I}^{(B)}$. The linear subspace of $W_{2}(3,4)$ composed of the elements of the type $C_{J}^{(A)}$ is denoted by $W_{J}$. The subspace $W_{I}$ is defined similarly.

Every $C_{J}^{(A)}$ is $O_{I}$-invariant and every $C_{I}^{(B)}$ is $O_{J}$-invariant because of (4.1). On the other hand it is written in page 357 of [3] that any element $C$ of $W_{2}(3,4)$ can be written in the form

$$
C=C_{J}^{(A)}+C_{I}^{(B)} .
$$

Thus, for example, $C$ is $J_{1}$-invariant if and only if $C_{J}^{(A)}$ is $J_{1}$-invariant.

$J_{1}$ is an element of the Lie algebra of $O_{J}$ and the infinitesimal action of $J_{1}$ on $C_{J}^{(A)}$ is given in page 356 of [3], or can be easily obtained from (4.3) and (4.4), in the form

$$
\begin{aligned}
& 4\left[C_{J}^{(A)}\left(J_{1} v, v, v, v ; w, w, w, w\right)+C_{J}^{(A)}\left(v, v, v, v ; J_{1} w, w, w, w\right)\right] \\
= & C_{J}^{\left(A^{\prime}\right)}(v, v, v, v ; w, w, w, w)
\end{aligned}
$$

where

$$
\begin{aligned}
A^{\prime \kappa \lambda \mu \nu}= & 2\left(\delta_{2}^{\kappa} A^{3 \lambda \mu \nu}+\delta_{2}^{\lambda} A^{3 \kappa \mu \nu}+\delta_{2}^{\mu} A^{3 \kappa \lambda \nu}+\delta_{2}^{\nu} A^{3 \kappa \lambda \mu}\right. \\
& \left.-\delta_{3}^{\kappa} A^{2 \lambda \mu \nu}-\delta_{3}^{\lambda} A^{2 \kappa \mu \nu}-\delta_{3}^{\mu} A^{2 \kappa \lambda \nu}-\delta_{3}^{\nu} A^{2 \kappa \lambda \mu}\right) .
\end{aligned}
$$

Thus $C$ is $J_{1}$-invariant if and only if $A^{\prime \kappa \lambda \mu \nu}$ vanish, hence

$$
A(x, y, z)=c\left(8 x^{4}-24 x^{2}\left(y^{2}+z^{2}\right)+3\left(y^{2}+z^{2}\right)^{2}\right)
$$

where $c$ is an arbitrary number [3].

In order to get a $J$-invariant $C$ where

$$
J=a_{1} J_{1}+a_{2} J_{2}+a_{3} J_{3}, \quad\left(a_{1}\right)^{2}+\left(a_{2}\right)^{2}+\left(a_{3}\right)^{2}=1,
$$

we take a rotation of $R^{3}$ such that

$$
\begin{aligned}
& ' x=a_{1} x+a_{2} y+a_{3} z, \\
& { }^{\prime} y=m_{1} x+m_{2} y+m_{3} z, \\
& { }^{\prime} z=n_{1} x+n_{2} y+n_{3} z .
\end{aligned}
$$


Then $C_{J}^{(B)}$ is $J$-invariant if and only if $B$ is given by

$$
B(x, y, z)=c\left[8\left({ }^{\prime} x\right)^{4}-24\left({ }^{\prime} x\right)^{2}\left(\left({ }^{\prime} y\right)^{2}+\left({ }^{\prime} z\right)^{2}\right)+3\left(\left({ }^{\prime} y\right)^{2}+\left({ }^{\prime} z\right)^{2}\right)^{2}\right],
$$

where $c$ is an arbitrary number. It is clear that $B$ depends only on $a_{1}, a_{2}, a_{3}$ on account of

$$
\left({ }^{\prime} y\right)^{2}+\left({ }^{\prime} z\right)^{2}=x^{2}+y^{2}+z^{2}-\left(a_{1} x+a_{2} y+a_{3} z\right)^{2} .
$$

Similarly an $I$-invariant $C$ is obtained if $I$ is given by

$$
I=a_{1} I_{1}+a_{2} I_{2}+a_{3} I_{3}, \quad\left(a_{1}\right)^{2}+\left(a_{2}\right)^{2}+\left(a_{3}\right)^{2}=1
$$

in the form $C=C_{I}^{(B)}$ where $B$ is given by (4.12).

Thus we get the following theorems (see [3]).

THEOREM 4.1. Let $J$ (resp. I) be given by (4.10) (resp. (4.13)). Then any $J$-invariant (resp. I-invariant) element $C$ of $W_{2}(m, s)$ is given by

$$
C=C_{I}^{(A)}+C_{J}^{(B)} \quad \text { resp. } C=C_{J}^{(A)}+C_{I}^{(B)}
$$

where $A$ is an arbitrary homogeneous harmonic polynomial in $R^{3}$ of degree 4 and $B$ is given by (4.11) and (4.12).

THEOREM 4.2. Let us take $J$ and I such that

$$
\begin{aligned}
& J=a_{1} J_{1}+a_{2} J_{2}+a_{3} J_{3}, \quad\left(a_{1}\right)^{2}+\left(a_{2}\right)^{2}+\left(a_{3}\right)^{2}=1, \\
& I=b_{1} I_{1}+b_{2} I_{2}+b_{3} I_{3}, \quad\left(b_{1}\right)^{2}+\left(b_{2}\right)^{2}+\left(b_{3}\right)^{2}=1
\end{aligned}
$$

and consider the subgroup $G$ of $\mathrm{SO}(4)$ generated by $J$ and $I$. Then any G-invariant $C$ is given by

$$
C=a C_{J}^{(A)}+b C_{I}^{(B)}
$$

where $a$ and $b$ are arbitrary numbers and

$$
\begin{aligned}
A(x, y, z)= & 8\left(a_{1} x+a_{2} y+a_{3} z\right)^{4}-24\left(a_{1} x+a_{2} y+a_{3} z\right)^{2} \\
& \times\left(x^{2}+y^{2}+z^{2}-\left(a_{1} x+a_{2} y+a_{3} z\right)^{2}\right) \\
+ & 3\left(x^{2}+y^{2}+z^{2}-\left(a_{1} x+a_{2} y+a_{3} z\right)^{2}\right)^{2}, \\
B(x, y, z)= & 8\left(b_{1} x+b_{2} y+b_{3} z\right)^{4}-24\left(b_{1} x+b_{2} y+b_{3} z\right)^{2} \\
& \times\left(x^{2}+y^{2}+z^{2}-\left(b_{1} x+b_{2} y+b_{3} z\right)^{2}\right) \\
+ & 3\left(x^{2}+y^{2}+z^{2}-\left(b_{1} x+b_{2} y+b_{3} z\right)^{2}\right)^{2} .
\end{aligned}
$$

DEFINITION 4.2. A one-parameter subgroup of $O_{J}$ is called a $J$-type subgroup of $\mathrm{SO}(4)$. Similarly an $I$-type subgroup is defined. A one-parameter subgroup of $\mathrm{SO}(4)$ which is not $J$-type nor $I$-type is called a mixed type subgroup.

As the dimension of the space of homogeneous harmonic polynomials in $R^{3}$ 
of degree 4 is 9 , we have the following theorem.

THEOREM 4.3. Let $k$ be a one-parameter subgroup of $\mathrm{SO}(4)$. Then there exist k-invariant elements of $W_{2}(3,4)$. If $k$ is of mixed type, then the set of $k$-invariant elements is a two-dimensional linear subspace of $W_{2}(3,4)$ spanned by a one-dimensional linear subspace of $W_{J}$ and a one-dimensional linear subspace of $W_{I}$. If $k$ is of J-type or of I-type, then the set of $k$-invariant elements is a ten-dimensional linear subspace of $W_{2}(3,4)$ containing $W_{I}$ or $W_{J}$.

Now let us take two mixed type one-parameter subgroups $k_{1}, k_{2}$ of $\mathrm{SO}(4)$ and let $G$ be the subgroup generated by $k_{1}, k_{2}$. The Lie algebra of $G$ is denoted by $g$. Taking a suitable coordinate system of $R^{3}$ we can consider that $\mathrm{g}$ is generated by one of the following pairs of elements of $\mathrm{g}$

(i) $J_{1}+a_{1} I_{1}+a_{2} I_{2}+a_{3} I_{3}, \quad b_{1} I_{1}+b_{2} I_{2}+b_{3} I_{3}$,

(ii) $J_{1}+a_{1} I_{1}+a_{2} I_{2}+a_{3} I_{3}, J_{2}+b_{1} I_{1}+b_{2} I_{2}+b_{3} I_{3}$,

(iii) $I_{1}+a_{1} J_{1}+a_{2} J_{2}+a_{3} J_{3}, \quad b_{1} J_{1}+b_{2} J_{2}+b_{3} J_{3}$,

(iv) $I_{1}+a_{1} J_{1}+a_{2} J_{2}+a_{3} J_{3}, \quad I_{2}+b_{1} J_{1}+b_{2} J_{2}+b_{3} J_{3}$.

But, in order to find the property of $G$, we need to consider only (i) and (ii).

We take vectors $a$ and $b$ of $R^{3}$ with components $\left(a_{1}, a_{2}, a_{3}\right)$ and $\left(b_{1}, b_{2}, b_{3}\right)$ respectively. We consider also that $I_{1}, I_{2}, I_{3}$ are the components of an imaginary vector $I$. Then we can write (i) and (ii) tersely in the form

(i) $\quad K_{1}=J_{1}+(a, I), \quad K_{2}=(b, I) \quad(a, b \neq 0)$,

(ii) $\quad K_{1}=J_{1}+(a, I), \quad K_{2}=J_{2}+(b, I) \quad(a \neq 0)$

where $($,$) is the inner product in R^{3}$. Let us use notations $[a, b]$ and $[a, b, c]$ used in vector algebra of $R^{3}$.

If we have $(\mathrm{i})^{\prime}$, then $\mathrm{g}$ contains $(c, I)$ where

$$
c=[a, b] \text {. }
$$

The simplest case occurs when the vectors $a$ and $b$ are not linearly independent. Then $g$ is spanned by $J_{1}$ and $(a, I)$. If $a$ and $b$ are linearly independent, we have $K_{3}=(c, I) \in \mathfrak{g}$ and

$$
\begin{aligned}
& \frac{1}{2}\left(K_{1} K_{3}-K_{3} K_{1}\right)=([a, c], I)=(a, b)(a, I)-(a, a)(b, I) \in \mathrm{g} \\
& \frac{1}{2}\left(K_{2} K_{3}-K_{3} K_{2}\right)=([b, c], I)=(b, b)(a, I)-(a, b)(b, I) \in \mathrm{g} .
\end{aligned}
$$

As we have $(a, a)(b, b)>(a, b)^{2}$ by assumption, we get $(a, I) \in \mathfrak{g},(b, I) \in \mathfrak{g}$, hence g contains $I_{1}, I_{2}, I_{3}$.

If we have (ii)', we define $K_{3}$ by

$$
K_{3}=\frac{1}{2}\left(K_{1} K_{2}-K_{2} K_{1}\right)=J_{3}+(c, I), \quad c=[a, b] .
$$


Then we have

$$
\begin{aligned}
& K_{1}-\frac{1}{2}\left(K_{2} K_{3}-K_{3} K_{2}\right)=(a, I)-[b, c, I], \\
& K_{2}-\frac{1}{2}\left(K_{3} K_{1}-K_{1} K_{3}\right)=(b, I)-[c, a, I],
\end{aligned}
$$

hence $\mathrm{g}$ contains

$$
\begin{aligned}
& K_{4}=(1-(b, b))(a, I)+(a, b)(b, I), \\
& K_{5}=(a, b)(a, I)+(1-(a, a))(b, I) .
\end{aligned}
$$

If $a$ and $b$ are not linearly independent, then $c=0$. Hence $g$ contains $J_{3}$ and consequently $J_{1}$ and $J_{2}$. Thus $g^{\prime}$ is spanned by $J_{1}, J_{2}, J_{3}$ and $(a, I)$.

Assume $a$ and $b$ are linearly independent. As we have

$$
\begin{aligned}
& K_{1} K_{4}-K_{4} K_{1}=2(a, b)(c, I), \\
& K_{1} K_{5}-K_{5} K_{1}=2(1-(a, a))(c, I),
\end{aligned}
$$

we get $(c, I) \in \mathfrak{g}$ except the case $(a, b)=0,(a, a)=1$. Similarly, from $K_{4} K_{2}-K_{2} K_{4}$ $=2(1-(b, b))(c, I)$ we get $(c, I) \in \mathfrak{g}$ if $(b, b) \neq 1$. If $(c, I) \in \mathfrak{g}$, then $\mathfrak{g}$ contains $(a, I)$ and $(b, I)$ and is spanned by $J_{1}, J_{2}, J_{3}, I_{1}, I_{2}, I_{3}$.

If

$$
(a, b)=0, \quad(a, a)=1, \quad(b, b)=1
$$

is satisfied, then $K_{4}$ and $K_{5}$ vanish. In this case $\mathfrak{g}$ is spanned by $J_{1}+(a, I)$, $J_{2}+(b, I), J_{3}+(c, I)$ where $[a, b]=c,[b, c]=a,[c, a]=b$.

To sum up we have the following cases.

(i. 1) $a$ and $b$ are not linearly independent. Then $g$ is spanned by $J_{1}$ and $(a, I)$.

(i. 2) $a$ and $b$ are linearly independent. Then $g$ is spanned by $J_{1}, I_{1}, I_{2}, I_{3}$. $(a, I)$.

(ii. 1) $a$ and $b$ are not linearly independent. Then $g$ is spanned by $J_{1}, J_{2}, J_{3}$,

(ii. 2) $a$ and $b$ are linearly independent and (4.18) is satisfied. Then $g$ is spanned by $J_{1}+(a, I), J_{2}+(b, I), J_{3}+(c, I)$.

(ii. 3) $a$ and $b$ are linearly independent and (4.18) is not satisfied. Then $g$ is spanned by $J_{1}, J_{2}, J_{3}, I_{1}, I_{2}, I_{3}$.

Thus we have the following theorem.

THEOREM 4.4. Let $G$ be the subgroup of $\mathrm{SO}(4)$ generated by two one-parameter subgroups $k_{1}, k_{2}$ of mixed type. Then there exist the following four cases.

( $\alpha) G$ is generated by a J-type one-parameter subgroup and an I-type oneparameter subgroup.

( $\beta$ ) $G$ is generated by a J-type one-parameter subgroup and $O_{I}$, or by an 
I-type one-parameter subgroup and $O_{J}$.

( $\gamma$ ) $G$ is generated by $J_{1}+(a, I), J_{2}+(b, I), J_{3}+(c, I)$ where $a$ and $b$ satisfy (4.18) and $c=[a, b]$.

(ס) $G=\mathrm{SO}(4)$.

If $(\gamma)$ or $(\boldsymbol{\delta})$ is the case, then no $G$-invariant elements of $W_{2}(3,4)$ exist except the trivial element 0 . If $(\alpha)$ is the case, $G$-invariant elements are obtained by Theorem 4.2. If $(\beta)$ is the case, the set of $G$-invariant elements is a onedimensional linear subspace of $W_{J}$ or of $W_{I}$ and can be obtained with the use of Theorem 4.1 .

\section{Some invariant e'ements of $W_{2}(m, s)$.}

Let $K$ be an element of $W_{2}(m, 4)$. Then $K(3,0)=0[2]$, [4]. The bi-symmetric tensor given by

$$
(C(s, 0))(a, b)=\sum_{p=0}^{\sigma-2} c_{p}\langle a, a\rangle^{p}\langle b, b\rangle^{p}\langle a, b\rangle^{s-2 p-4}(K(4,0))(a, b),
$$

where $\sigma=[s / 2]$ and $c_{0}, c_{1}, \cdots, c_{\sigma-2}$ satisfy

$$
2 p(m+2 s-2 p-1) c_{p}+(s-2 p-2)(s-2 p-3) c_{p-1}=0,
$$

is an element of $W_{\mathbf{2}}(m, s)$. We have the following theorem.

THEOREM 5.1. Let $k$ be a subgroup of $\mathrm{SO}(m+1)$ and $K$ be k-invariant. Then $C$ is also k-invariant.

Proof is easy as $\langle g a, g b\rangle=\langle a, b\rangle$ for $g \in \mathrm{SO}(m+1)$.

Remark. It is also easy to prove that $C$ belongs to $W_{2}(m, s) . \quad C$ is bi-symmetric and satisfies $C(2,0)=0$. That $C$ is harmonic is assured by (5.2).

Definition 5.1. Let $C$ be an element of $W_{2}(m, s)$. The bi-symmetric tensor $C^{1}$ of bi-degree $(s-1, s-1)$ given by

$$
C^{1}(s-1,0)=\sum_{i} C\left(a, \cdots, a, e_{\imath} ; b, \cdots, b, e_{\imath}\right)
$$

is called the first contraction of $C$.

LEMMA 5.2. $\quad C^{1}$ is an element of $W_{2}(m, s-1)$.

Proof. As $C$ is harmonic, $C^{1}$ is also harmonic. On the other hand, from $C(a, a, b, \cdots, b ; b, \cdots, b)=0$, we get

$$
\Sigma_{i} C\left(a, a, b, \cdots, b, e_{\imath} ; b, \cdots, b, e_{\imath}\right)=0,
$$

hence $C^{1}(a, a, b, \cdots, b ; b, \cdots, b)=0$. 
THEOREM 5.3. Let $k$ be a subgroup of $\mathrm{SO}(m+1)$ and $C$ be a k-invariant element of $W_{2}(m, s)$. Then $C^{1}$ is also k-invariant. basis.

Proof is easy as $\left\{g e_{1}, \cdots, g e_{m+1}\right\}$, where $g \in \mathrm{SO}(m+1)$, is also an orthonormal

\section{Geodesics.}

We consider geodesics in isometric minimal immersions $f: S^{3}(1) \rightarrow S^{24}(r)$, $r^{2}=1 / 8$, namely the case $m=3, s=4$. A geodesic $\gamma$ in $S^{3}(1)$ can be written

$$
u(t)=a \cos t+b \sin t
$$

where $a$ and $b$ are orthonormal vectors in $R^{4}$ and $t$ is a parameter such that $u^{\prime}=d u / d t$ is a unit vector. $\Gamma=i \circ f(\gamma)$ is a geodesic in the image $i \circ f\left(S^{3}(1)\right)$ and the unit tangent vector of $\Gamma$ is $i_{1}^{A} \tilde{e}_{A}$ where $\imath_{1}^{A}=d F^{A}(u, u, u, u) / d t=4 F^{A}\left(u, u, u, u^{\prime}\right)$.

Let us define $F_{p}^{A}(p=0,1,2,3,4)$ by $F_{p}^{A}=F^{A}\left(u, \cdots, u^{\prime}\right)$ where $p$ of $u$ in $F^{A}(u, u, u, u)$ are replaced with $u^{\prime}$. Then, as we have $u^{\prime \prime}=-u$, we get

$$
d\left(F_{p}^{A}\right) / d t=-p F_{p-1}^{A}+(4-p) F_{p+1}^{A},
$$

hence

$$
\begin{aligned}
& \left(F_{0}^{A}\right)^{\prime \prime}=-4 F_{0}^{A}+12 F_{2}^{A}, \\
& \left(F_{0}^{A}\right)^{(3)}=-40 F_{1}^{A}+24 F_{3}^{A}, \\
& \left(F_{0}^{A}\right)^{(4)}=40 F_{0}^{A}-192 F_{2}^{A}+24 F_{4}^{A}, \\
& \left(F_{0}^{A}\right)^{(5)}=544 F_{1}^{A}-480 F_{3}^{A}=-64\left(F_{0}^{A}\right)^{\prime}+20\left(F_{0}^{A}\right)^{(3)} .
\end{aligned}
$$

Let us define $C_{p, q}$ and $U_{p, q}$, which are obtained from $C(u, u, u, u ; u, u, u, u)$ and $U(u, u, u, u ; u, u, u, u)$ when some of $u$ are replaced with $u^{\prime}$, by

$$
\begin{aligned}
& C_{p, q}\left(u, u^{\prime}\right)=C\left(u, \cdots, u^{\prime} ; u, \cdots, u^{\prime}\right), \\
& U_{p, q}\left(u, u^{\prime}\right)=U\left(u, \cdots, u^{\prime} ; u, \cdots, u^{\prime}\right)
\end{aligned}
$$

where in the right hand side of each formula $u^{\prime}$ appears $p$ times before the semicolon and $q$ times after the semicolon. As it is written in page 347 of [3], $C$ satisfies

$$
C_{4,0}=-4 C_{3,1}=6 C_{2,2}
$$

and $C_{p, q}=0$ if $p+q \neq 4$, and this leads to $d C_{p, q} / d t=0$. On the other hand, as $U$ is given by (3.1), $U_{p, q}$ is a constant depending only on $p$ and $q$, and especially $U_{p, q}=0$ if $p+q$ is an odd number.

The relation between $C_{p, q}, U_{p, q}$ and $F_{p}^{A}, F_{q}^{A}$ is, as it is obtained from (1.3) and (3.2),

$$
\Sigma_{A} F_{p}^{A} F_{q}^{A}=C_{p, q}\left(u, u^{\prime}\right)+c^{\prime} U_{p, q}\left(u, u^{\prime}\right) .
$$


This proves that $\Sigma_{A} F_{p}^{A} F_{q}^{A}$ does not depend on $t$, but depends only on $p, q$ and $C(a, a, a, a ; b, b, b, b)$.

The Frenet formula of $\Gamma$ is written in the form

$$
\begin{aligned}
& i_{1}^{A}=\left(F_{0}^{A}\right)^{\prime}, \\
& \left(i_{1}^{A}\right)^{\prime}=k_{1} i_{2}^{A}, \\
& \left(i_{2}^{A}\right)^{\prime}=-k_{1} i_{1}^{A}+k_{2} i_{3}^{A}, \\
& \left(i_{3}^{A}\right)^{\prime}=-k_{2} i_{2}^{A}+k_{3} i_{4}^{A}, \\
& \left(i_{4}^{A}\right)^{\prime}=-k_{3} i_{3}^{A} .
\end{aligned}
$$

First, we have

$$
\begin{aligned}
\left(k_{1}\right)^{2} & =\Sigma_{A}\left(\left(F_{0}^{A}\right)^{\prime \prime}\right)^{2} \\
& =16 \Sigma_{A}\left(F_{0}^{A}\right)^{2}-96 \Sigma_{A} F_{0}^{A} F_{2}^{A}+144 \Sigma_{A}\left(F_{2}^{A}\right)^{2},
\end{aligned}
$$

and this proves that $\left(k_{1}\right)^{\prime}=0$. Next we get

hence

$$
\left(F_{0}^{A}\right)^{(3)}=\left(i_{1}^{A}\right)^{\prime \prime}=k_{1}\left(i_{2}^{A}\right)^{\prime}=-\left(k_{1}\right)^{2} i_{1}^{A}+k_{1} k_{2} i_{3}^{A},
$$

$$
\left(k_{1} k_{2}\right)^{2}+\left(k_{1}\right)^{4}=\Sigma_{A}\left(\left(F_{0}^{A}\right)^{(3)}\right)^{2},
$$

which proves $\left(k_{2}\right)^{\prime}=0$. In this way we also get $\left(k_{3}\right)^{\prime}=0$, hence the following theorem.

THEOREM 6.1. Every geodesic $\Gamma$ in the image $i \circ f\left(S^{3}(1)\right)$ has constant curvatures $k_{1}, k_{2}, k_{3}$ which depend on the choice of the geodesic.

We can compute straightforwardly the curvatures $k_{1}, k_{2}, k_{3}$ from the Frenet formula, (6.2) and (6.3) in detail and get as a result the following theorem.

THEOREM 6.2. The curvatures $k_{1}, k_{2}, k_{3}$ of any geodesic $\Gamma=i \circ f(\gamma)$ are constants which depend only on $C(a, a, a, a ; b, b, b, b)$ if $\gamma$ is given by (6.1) and $C$ is the element of $W_{2}(3,4)$ associated with $f$.

Let $g$ be an element of $\mathrm{SO}(4)$ and take another geodesic $\hat{\gamma}=g \gamma$, where we have $\hat{u}(t)=g a \cos t+g b \sin t$. Then, as a result of Theorem 6.2, we find that the curvatures $\hat{k}_{1}, \hat{k}_{2}, \hat{k}_{3}$ satisfy $\hat{k}_{1}=k_{1}, \hat{k}_{2}=k_{2}, \hat{k}_{3}=k_{3}$ if $g^{-1} C=C$.

Thus we have the following theorem.

THEOREM 6.3. Let $f$ be an isometric minimal immersion $S^{3}(1) \rightarrow S^{24}(r), r^{2}=1 / 8$, such that the element $C$ associated with $f$ is g-invariant where $g$ is an element of $\mathrm{SO}(4)$. Then, for any geodesic $\gamma$ of $S^{3}(1)$, the geodesics $i \circ f(\gamma)$ and $i \circ f(g \gamma)$ have the same set of curvatures $k_{1}, k_{2}, k_{3}$.

As the trivial element 0 of $W_{2}(3,4)$ is $\mathrm{SO}(4)$-invariant, this theorem also 
proves that a standard minimal immersion is a helical immersion [5], [6].

Let $J$ be given by (4.14). When $P$ is a point of $S^{3}(1)$ we say that the locus $\left\{e^{J t} P, t \in R\right\}$ is a $J$-orbit. As any $J$-orbit is a geodesic and carried into a $J$-orbit by any element of $O_{I}$, we get the following theorem.

THEOREM 6.4. Let $J$ be given by (4.14), $f$ be an isometric minimal immersion $S^{3}(1) \rightarrow S^{24}(r), r^{2}=1 / 8$, subject to $C_{J}^{(A)}$ where $A$ is given by (4.16) and let $k_{1}, k_{2}, k_{3}$ be the curvatures of the geodesic $\Gamma=i \circ f(\gamma)$ of $i \circ f\left(S^{3}(1)\right)$ where $\gamma$ is the J-orbit passing a point a of $S^{3}(1)$. Then each of these curvatures is independent of the choice of the point a.

The theorem is valid when we take $I$ instead of $J$.

\section{REFERENCES}

[1] M. Do Carmo and N. Wallach, Minimal immersions of spheres into spheres, Ann. of Math., (2) 93 (1971), 43-62.

[2] Y. Mutō, Some properties of isometric minimal immersions of spheres into spheres, Kodai Math. J., 6 (1983), 308-332.

[3] Y. Mutō, The space $W_{2}$ of isometric minimal immersions of the three-dimensional sphere into spheres, Tokyo J. of Math., 7 (1984), 337-358.

[4] Y. MuTō, Isometric minimal immersions of spheres into spheres isotropic up to some order, Yokohama Math. J., 32 (1984), 159-180.

[5] K. SaKamoto, Helical immersions into a unit sphere, Math. Ann., 261 (1982), 63-80.

[6] K. TsuKadA, Isotropic minimal immersions of spheres into spheres, J. Math. Soc. Japan, 35 (1983), 355-379.

34-6-904, 5-С HOME,

KOISHIKAWA, BUNKYO-KU,

TOKYO 112 , JAPAN 